\title{
Vandermonde-Cocyclic Codes and a suitable DFT
}

\author{
Kiran .T and B. Sundar Rajan \\ Dept. ECE, Indian Institute of Science, India \\ $\{$ kirant@protocol., bsrajan@\}ece.iisc.ernet.in
}

\begin{abstract}
A new transform is defined for the class of cocyclic group ring codes over a Galois ring, where the underlying group is Abelian and the cocycle is the Vandermonde-cocycle on the Abelian group. The class of Vandermonde-cocyclic (VC) group ring codes are characterized using this new transform by means of a structure theorem for $\mathrm{VC}$ group rings.

For a prime $p$, let $G R\left(p^{a}, l\right)$ denote the Galois ring with characteristic $p^{a}[1]$. Let $\mathcal{T}_{a, l}^{*}$ denote the unique cyclic subgroup of order $p^{l}-1$ in the group of units $G R\left(p^{a}, l\right)^{*}$. Let $G$, a finite Abelian group (with $|G|=\mathrm{n}$ ), be a direct product of $r$ cyclic subgroups denoted by $C_{r-1}, \ldots, C_{0}$ of orders $m_{r-1}, \ldots, m_{0}$ respectively. Any element $g \in G$ can be denoted as $g_{i}$ or $g_{\lceil i\rceil}$, where $\lceil i\rceil=\left\lceil i_{r-1}, i_{r-2}, \cdots, i_{0}\right\rceil$ is the mixed-radix representation of $i \in I_{n}=\{0, \ldots, n-$ $1\}$ using $m_{r-1}, m_{r-2}, \ldots, m_{0}$ as the mixed-radixes and the group operation of $G$ can also be specified using the mixedradix addition [2]. We consider linear codes of length $n=m_{r-1} \ldots m_{1} m_{0}$ over the Galois ring $G R\left(p^{a}, l\right)$ such that $\operatorname{gcd}(p, n)=1$. We use $q$ for $p^{l}$ for notational simplicity.

A function $\psi: G \times G \rightarrow \mathcal{T}_{a, l}^{*}$ is called a cocycle on $G$ if $\psi(x, y) \psi(x y, z)=\psi(y, z) \psi(x, y z)$ for all $x, y, z \in G$ [3]. Let $\bar{\beta}=\left(\beta_{r-1}, \beta_{r-2}, \ldots, \beta_{0}\right)$, where each $\beta_{\lambda}$ is an element of $\mathcal{T}_{a, l}^{*}$, such that $d_{\lambda}$ (the order of $\beta_{\lambda}$ ) divides $m_{\lambda}$ for all $\lambda=0,1, \ldots, r-1$. The map on $G \times G$ defined as $\psi_{\bar{\beta}}\left(g_{\lceil i\rceil}, g_{\lceil j\rceil}\right)=\prod_{\lambda=0}^{r-1} \beta_{\lambda}^{i_{\lambda} j_{\lambda}}$, for $\lceil i\rceil=\left\lceil i_{r-1}, i_{r-2}, \ldots, i_{0}\right\rceil$ and $\lceil j\rceil=\left\lceil j_{r-1}, j_{r-2}, \ldots, j_{0}\right\rceil$, is a cocycle. We call this cocycle as Vandermonde-cocycle on an Abelian group. For any cocycle $\psi$ on $G$, let $G R\left(p^{a}, l\right)^{\psi}[G]$ be the twisted group ring which has the same module structure as the group ring $G R\left(p^{a}, l\right)[G]$, but the multiplication is twisted, which is a linear extension of $g . h=\psi(g, h) g h$ for all $g, h \in G$. A cocyclic group ring code is an ideal in $G R\left(p^{a}, l\right)^{\psi}[G]$. Vandermonde-cocyclic (VC) group ring codes are ideals in the cocyclic group ring $G R^{\psi} \bar{\beta}\left(p^{a}, l\right)[G]$. Let $m$ be the smallest positive integer such that $m_{\lambda} \mid\left(q^{m}-1\right)$ and also $2 d_{\lambda} \mid\left(q^{m}-1\right)$ for all $\lambda=0,1, \ldots, r-1$. Let $\alpha_{\lambda}=\zeta^{s_{\lambda}}$ be a primitive root of unity of order $m_{\lambda}$ and $\gamma_{\lambda}=\zeta^{h_{\lambda}}$ be a square-root of $\beta_{\lambda}$, for all $\lambda$, belonging to $\mathcal{T}_{a, l m}^{*}=\langle\zeta\rangle \subset G R\left(p^{a}, l m\right)$.
\end{abstract}

Definition 1 (VC-DFT) Let $\vec{a}=\left(a_{0}, a_{1}, \ldots, a_{n-1}\right) \in$ $G R\left(p^{a}, l\right)^{n}$ and let $\alpha_{\lambda}$ and $\gamma_{\lambda}$ be as defined above. The $V C$ DFT vector $\vec{A}=\left(A_{0}, A_{1}, \ldots, A_{n-1}\right) \in G R\left(p^{a}, l m\right)^{n}$ of $\vec{a}$ is defined as $A_{j}=\sum_{i=0}^{n-1}\left(\prod_{\lambda=0}^{r-1} \gamma_{\lambda}^{-i_{\lambda}^{2}} \alpha_{\lambda}^{i_{\lambda} j_{\lambda}}\right) a_{i}$ for all $j \in I_{n}$, where $i=\left\lceil i_{r-1}, i_{r-2}, \cdots, i_{0}\right\rceil$ and $j=\left\lceil j_{r-1}, j_{r-2}, \cdots, j_{0}\right\rceil$ are mixed-radix representations of $i$ and $j$.

\section{Lemma 1 (Properties of VC-DFT)}

$$
\left(\prod_{\lambda=0}^{r-1} \beta_{\lambda}^{i_{\lambda}}\right) a_{i \ominus\lceil 1,1, \ldots, 1\rceil} \stackrel{v c-d f t}{\longleftrightarrow}\left(\prod_{\lambda=0}^{r-1} \gamma_{\lambda} \alpha_{\lambda}^{j_{\lambda}}\right) A_{j},
$$

\footnotetext{
${ }^{1}$ This work was partly funded by the DRDO-IISc Program on Advanced Research in Mathematical Engineering through a grant to B.S.Rajan.
}

$$
\begin{aligned}
& c_{i}=\sum_{k=0}^{n-1} a_{i \ominus k} b_{k} \psi_{\bar{\beta}}\left(g_{i \ominus k}, g_{k}\right) \stackrel{v c-d f t}{\longleftrightarrow} C_{j}=B_{j} A_{j}, \\
& \left(\prod_{\lambda=0}^{r-1} \alpha_{\lambda}^{i_{\lambda}}\right) a_{i} \stackrel{v c-d f t}{\longleftrightarrow} A_{j \oplus\lceil 1,1, \ldots, 1\rceil}, \\
& c_{i}=a_{i} b_{i} \stackrel{v c-d f t}{\longleftrightarrow} \frac{1}{n} \sum_{k=0}^{n-1} B_{k} A_{j \ominus k}^{(u)}
\end{aligned}
$$

where $A_{k}^{(u)}=\sum_{i=0}^{n-1}\left(\prod_{\lambda=0}^{r-1} \alpha_{\lambda}^{i_{\lambda} k_{\lambda}}\right) a_{i}$ is the Generalized DFT for Abelian codes [2] and $\oplus, \ominus$ denote the mixed-radix addition and subtraction respectively.

Let $\sigma_{0}$ denote the Frobenius automorphism of $G R\left(p^{a}, \operatorname{lm}\right)$ and let $\sigma=\sigma_{0}^{l}$. A vector $\vec{A} \in G R\left(p^{a}, l m\right)^{n}$ is the VCDFT vector of $\vec{a} \in G R\left(p^{a}, l\right)^{n}$, if and only if $\sigma^{k}\left(A_{j}\right)=A_{i}$ where $i=\left\lceil i_{r-1}, i_{r-2}, \ldots, i_{0}\right\rceil$ is such that, $i_{\lambda}=\phi_{\lambda}^{k}\left(j_{\lambda}\right)=$ $\left(\frac{-\left(q^{k}-1\right) h_{\lambda}}{s_{\lambda}}+q^{k} j_{\lambda}\right)$ modulo $m_{\lambda}$ for all $\lambda$. This is the conjugate symmetry property. Let $\Phi^{k}$ denote the mapping from $I_{n}$ to $I_{n}$, which maps $i=\left\lceil i_{r-1}, \ldots, i_{0}\right\rceil$ to $\Phi^{k}(i)=$ $\left\lceil\phi_{r-1}^{k}\left(i_{r-1}\right), \ldots, \phi_{0}^{k}\left(i_{0}\right)\right\rceil$. For every $j \in I_{n}$, the set $\widehat{\lceil j\rceil}=$ $\left\{j, \Phi^{1}(j), \Phi^{2}(j), \ldots, \Phi^{e_{j}-1}(j)\right\}$ where $e_{j}$ is the smallest integer such that $\Phi^{e_{j}}(j)=j$, is called the cyclotomic coset containing $\lceil j\rceil$ and, $e_{j}$ is called the exponent of $\lceil j\rceil$. Let $\mathbf{L} \subseteq I_{n}$ be the set containing one element from each of the cyclotomic cosets. The set of transform components $\widehat{A}_{\lceil i\rceil}=\left\{A_{\lceil j\rceil} \mid\lceil j\rceil \in \widehat{\lceil i\rceil}\right\}$ will be called the conjugacy class containing $A_{\lceil i\rceil}$. For a code $\mathcal{C}$ over $G R\left(p^{a}, l\right)$, let $\mathbf{C}_{\mathbf{j}}=\left\{A_{j} \mid \forall \vec{a} \in \mathcal{C}\right\}$ denote the set of distinct values taken by the $j$-th transform component of all the codewords in $\mathcal{C}$ and let $\mathbf{C}_{\mathbf{i}, \mathbf{j}}=\left\{\left(A_{i}, A_{j}\right) \mid \forall \vec{a} \in \mathcal{C}\right\}$.

Theorem 1 (Structure theorem) For any integer $n=$ $m_{r-1} m_{r-2} \ldots m_{0}$ and $\operatorname{gcd}(p, n)=1, G R\left(p^{a}, l\right)^{n}$ is isomorphic to $\bigoplus_{i \in \mathbf{L}} G R\left(p^{a}, l e_{i}\right)$. Further, a $\bar{\beta}-V C$ group ring code $\mathcal{C}$ over $\operatorname{GR}\left(p^{a}, l\right)$ is isomorphic to an ideal $\bigoplus_{i \in \mathbf{L}} \mathbf{C}_{\mathbf{i}}$, where $\mathbf{C}_{\mathbf{i}}=p^{\eta_{i}} G R\left(p^{a}, l e_{i}\right)$ for some fixed value of $\eta_{i}, 0 \leq \eta_{i} \leq a$ and transform components belonging to different conjugacy classes take values independently.

Here, $A_{i}$ and $A_{j}$ take values independently implies $\mathbf{C}_{\mathbf{i}, \mathbf{j}}=$ $\mathbf{C}_{\mathbf{i}} \times \mathbf{C}_{\mathbf{j}}$.

Transform Domain Characterization: A code over Galois ring $G R\left(p^{a}, l\right)$ is $\bar{\beta}$-VC iff the VC-DFT vector of each codeword satisfies the conjugate symmetry property and for any $i \in I_{n}$, $\mathbf{C}_{\mathbf{i}}=p^{\eta_{i}} G R\left(p^{a}, l e_{i}\right)$ for some fixed value of $\eta_{i}, 0 \leq \eta_{i} \leq a$.

\section{REFERENCES}

[1] McDonald B.R, Finite Rings with Identity, Marcel Dekker,Inc., New York, 1974.

[2] Kiran.T and B. Sundar Rajan, "Abelian codes over Galois rings closed under certain permutations," IEEE Trans. Inform. Theory, vol. 49, no. 9, Sept 2003.

[3] K.J. Horadam and A.A.I. Perera, "Codes from cocycles," in $A A E C C-12$, T. Mora and H. Mattson, Eds., 1997, LNCS 1255, pp. $151-163$. 